\title{
Comparison of VLF Wave Activity in the Solar Wind During Solar Maximum and Minimum: Ulysses Observations
}

\author{
Naiguo Lin, P. J. Kellogg, \\ University of Minnesota, Minneapolis, Minnesota, USA \\ R. J. MacDowall, \\ NASA Goddard Space Flight Center, Greenbelt, Maryland, USA \\ D. J. McComas, \\ Southwest Research Institute, San Antonio, Texas, USA \\ A. Balogh, and R. J. Forsyth \\ The Blackett Laboratory, Imperial College, London, UK
}

\begin{abstract}
We have compared observations of VLF waves ( 0.2 to $448 \mathrm{~Hz}$ ) made by Ulysses during its second fast latitude scan (near the solar maximum) with the wave observations during the first fast latitude scan in 1995, when the solar activity was approaching a minimum. The occurrences and properties of the waves are found to be similar during the solar maximum and solar minimum periods for slow and intermediate speed solar wind. The maximum intensity of the electromagnetic waves for the two solar cycle periods is comparable. These similarities suggest that the plasma conditions for the waves' excitation are similar for the slow and intermediate solar wind in both solar maximum and minimum phases. It is also found that the electric field noise detected in the low band channels, which are measuring less than $9 \mathrm{~Hz}$ signals, is contaminated by the spin modulation of the electric field due to photoelectrons around the spacecraft, especially when the ambient plasma density is low. This noise increases with increasing solar aspect angle.
\end{abstract}

\section{INTRODUCTION}

Observations of VLF waves made by the Unified Radio and Plasma Wave Experiments (URAP) of Ulysses during its first orbit, which occurred when the solar activity was approaching minimum, have found that there are different patterns and properties of wave activity occurring in the slow to intermediate $(\sim 300$ to $600 \mathrm{~km} / \mathrm{s})$ solar wind and in the fast solar wind $(1,2)$. During the solar minimum period, the slow to intermediate solar wind was observed at low and midlatitudes, i.e. within the heliospheric current sheet region. In this region enhanced electromagnetic waves in the whistler mode frequency range were observed near interplanetary (IP) shocks, during heliospheric current sheet crossings, and in other solar wind turbulence. These waves were most intense in compression regions of high-speed stream interfaces, which were observed in periods of increasing solar wind velocity. Electromagnetic whistler mode waves in the solar wind have been suggested to be excited by the whistler heat-flux instability and to be responsible for electron heat flux regulation in the solar wind [cf. (3) and references therein]. Comparison of the electron heat flux data with wave activity, however, is not conclusive: high levels of electron heat flux are often absent during periods of intense, low frequency electric wave activity (4).

Lin et al. (1) have also reported that enhanced electric fluctuations with little or no magnetic fluctuations are often observed at low band channels 
of the wave form analyzer (WFA) of the URAP instrument. The central frequencies of these channels range from 0.2 to $5.3 \mathrm{~Hz}$. These electric enhancements often occur in the expanding solar wind, i.e., in intervals when the measured solar-wind velocity is decreasing. The intensity of magnetic waves decreased dramatically in these intervals, indicating that these intervals did not favor the excitation of the whistler mode. As will be addressed below, the electric field enhancements seen in the low band channels contain electric field variations caused by photo-electron clouds around the spacecraft.

Outside the heliospheric current sheet region, in relatively stable fast solar wind streams, Ulysses observed nearly continuous electric wave activity with peak power near the local electron gyrofrequency, $f_{c e}$, (a few tens $\mathrm{Hz}$ ). These waves may also have a magnetic component but their levels have decreased below the search coil background at distances beyond $\sim 2.5$ AU.

The Ulysses mission has extended the observations to a full solar cycle. As Ulysses started the second orbit, the Sun was approaching maximum activity conditions. In this period, the spacecraft observed an irregularly structured mixture of slow and intermediate-speed solar wind and no entry into the fast solar wind (5). Strong and frequent wave activity has been observed, not only near the ecliptic plane and at mid-latitudes, but also at high latitudes. The plasma conditions of slow and intermediate solar wind existing at all latitudes during the second orbit, are responsible for the lack of latitudinal variations of the wave activity. The features of VLF wave activity described above, which were seen in low and midlatitudes during the solar minimum mission, were also observed at high latitudes during solar maximum. This paper presents the observations of VLF waves made during the second fast latitude scan, and compares the observations with those obtained during the period when Ulysses was in the slow to intermediate solar wind in the first fast latitude scan in 1995.

\section{OBSERVATIONS AND COMPARISON}

The second fast latitude scan occurred throughout the year 2001. Figure 1 displays the observations for days $100-170$ of 2001 , at heliographic latitudes $\sim-20^{\circ}$ to $27^{\circ}$. The top and bottom panels show the electric field and magnetic field spectra using 15-min averaged data. The wave power is expressed by the relative intensity, which is the measured signal minus the background noise of the instrument, divided by the background noise (1). A local $f_{c e}$ line is overplotted in each panel of the spectra.

The figure shows similar features of wave activity as those observed in the heliospheric current sheet region during the first orbit. The peak power of the wave activity is below $f_{c e}$, which indicates the waves are in the whistler mode frequency range. Electromagnetic waves, which are observed in both electric and magnetic field spectra, occur near interplanetary shocks (marked by the vertical dashed lines in the middle two panels) and where there is an increase in the solar wind velocity. These waves are seen most clearly in the high band channels $(>9 \mathrm{~Hz})$. In expanding solar wind stream regions, i.e. during the intervals when the solar wind velocity is decreasing, magnetic noise reduces significantly. The above features have been observed during the second orbit (starting at the beginning of the year 1998) at all latitudes. Electric waves are frequently observed in lower band data $(<9 \mathrm{~Hz})$. We notice that these are periods with low plasma density, as seen in the third panel where the electron density (calculated as $\mathrm{N}_{\mathrm{p}}+2 \mathrm{~N}_{\alpha}$, where $\mathrm{N}_{\mathrm{p}}$ and $\mathrm{N}_{\alpha}$ are the proton and helium ion density, respectively) is plotted. Some clear examples of these periods are marked with the yellow stripes in the middle two panels. The anti-correlation between the electric field enhancements and the electron density has led us to believe that the enhancements of the electric field are caused by photoelectron clouds around the spacecraft. This effect becomes stronger when the solar aspect angle (the angle of Sun-Ulysses-Earth) becomes larger in the second latitude scan than in the first.

In order to compare the intensity of the waves occurring during solar maximum and minimum periods, we compare the observations of VLF waves taken in the first and second latitude scans for a heliographic latitude range of $-20^{\circ}$ to $20^{\circ}$, where Ulysses was traveling in the slow to intermediate solar wind, at similar distances from the Sun for both scan periods. Observations for the first latitude scan in 1995 have been reported in paper (1). Figure 2 shows the comparison of spin plane magnetic and electric signals measured at the channel with a central frequency of 14 $\mathrm{Hz}$, which is typical for whistler mode waves observed in the solar wind at these distances. The first panel displays the power density of magnetic waves vs. heliographic latitude. In general, the maximum intensity of the waves in the second latitude scan in 2001 (red line) is comparable to that in the first scan (green line) in 1995, except for the strong enhancement of the wave on day 130, 2001, at a latitude of $\sim-5^{\circ}$, which is associated with a strong IP 


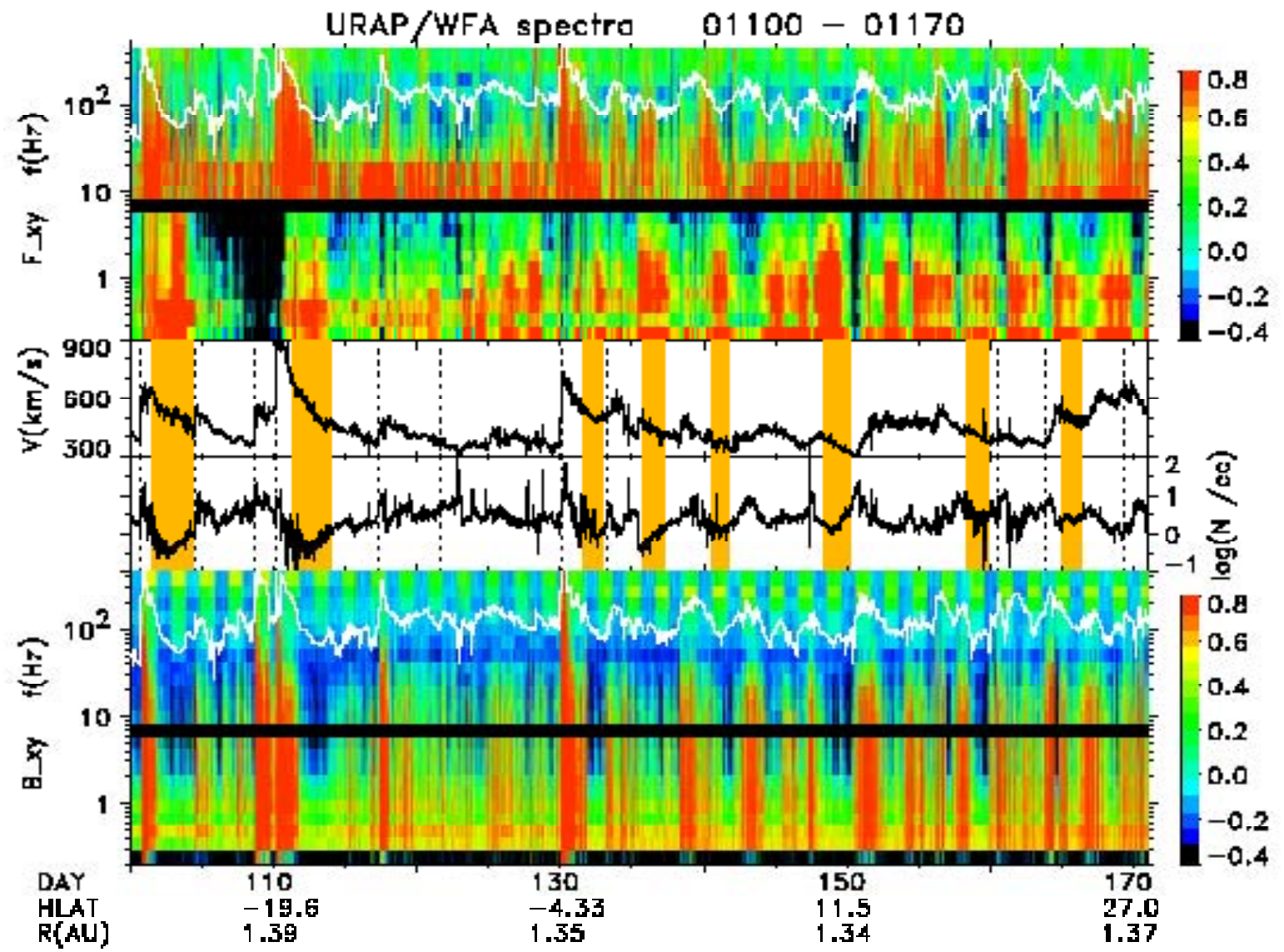

FIGURE 1. From top to bottom: Spectra of 15-min averaged relative intensity of spin plane electric wave power for the period from day 100 to day 170 of 2001 (the local $f_{c e}$ is overplotted as a white line); the solar wind velocity in $\mathrm{km} / \mathrm{s}$; the electron density; and the magnetic wave power in the same format as that of the electric field spectra. The vertical dotted lines mark IP shock crossings. The vertical yellow stripes in the two middle panels indicate periods of low-density level.

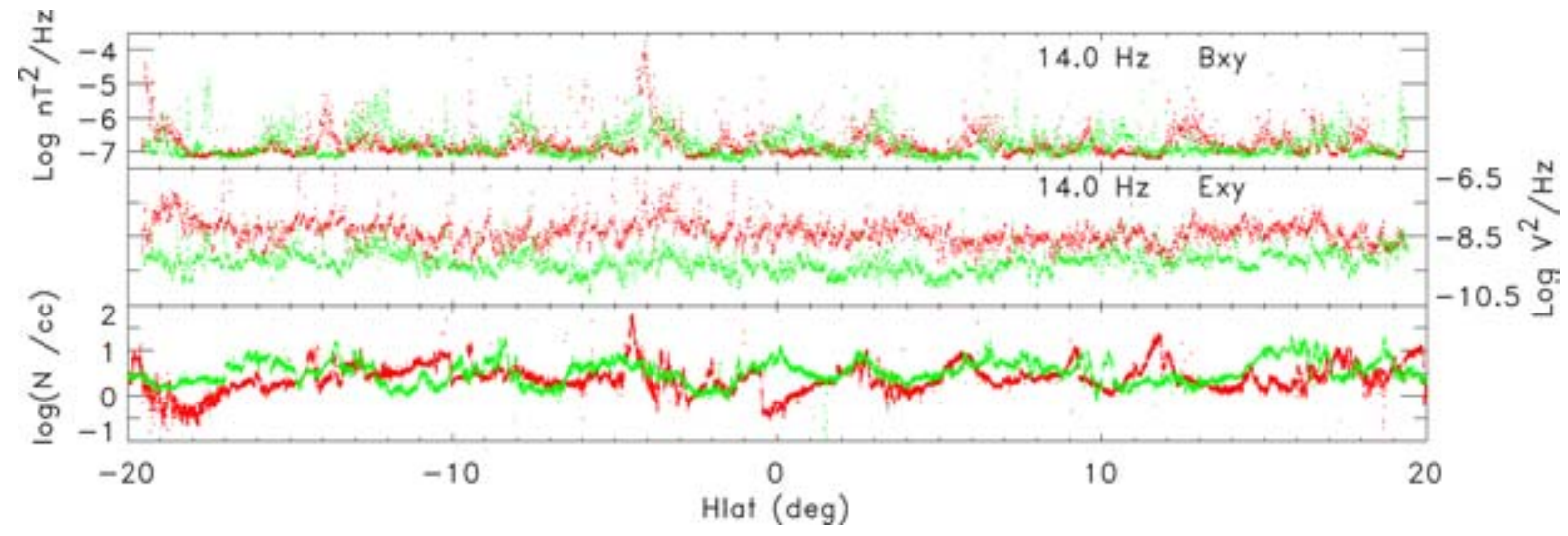

FIGURE 2. From top to bottom: The power density of spin plane magnetic field waves (in the log scale) at $14.0 \mathrm{~Hz}$ for the first fast latitude scan in 1995 (green line) and for the second latitude scan in 2001 (red line), plotted vs. heliographic latitude; the same as the first panel but for the electric field power density; the electron density calculated as $\mathrm{N}_{\mathrm{p}}+2 \mathrm{~N}_{\alpha}$.

shock. The second panel displays the comparison of electric wave power (expressed in $\mathrm{V}^{2} / \mathrm{Hz}$ ), which shows that the electric waves in the second scan (during the solar maximum period) are apparently 
slightly stronger than those in the first scan (during the solar minimum period). The electric measurements of Ulysses are affected by the variation of the solar aspect angle, with larger angles producing higher instrument background noise (1). The effect mainly exists in the low band channels $(<9 \mathrm{~Hz})$, while the high band channels are less affected. During the second latitude scan, the solar aspect angle for the period displayed was higher $\left(\sim 25^{\circ}-40^{\circ}\right)$ than that during the first latitude scan $\left(<13^{\circ}\right)$, which may contribute to the difference in the intensity for both periods. Latitude variations of the electron density for the two fast scan periods are plotted in the third panel, which shows the density varies in about the same range for both periods, indicating similar plasma conditions for the regions examined during solar maximum and minimum. In summary, our observations have shown that during the ascending phase of the solar cycle, when Ulysses was embedded in the slow and intermediate speed solar wind at all latitudes, and frequently encountered enhanced solar wind turbulence, interplanetary shocks, and current sheet crossings, the spacecraft observed VLF wave activity with an occurrence pattern similar to that observed in the low and mid-latitudes during the declining phase of the solar cycle (1). The intensity of the electromagnetic whistler mode waves, which are very commonly observed in the solar wind, is comparable for the two periods of different solar cycle phases. These similarities suggest that the plasma conditions for the waves' excitation are similar for the slow and intermediate solar wind in both solar cycle phases.

\section{THE PHOTOELECTRON EFFECT}

Electric field measurements in low band channels $(<9 \mathrm{~Hz})$ of the WFA are contaminated by the spinmodulated signal produced by the asymmetric spacecraft photoelectron cloud. This one cycle per spin signal leaks into all of the low band channels, because the 16-point Walsh transform for the low band data does not have sufficient resolution. This effect is stronger when the ambient electron density decreases, and thus produces an apparent anti-correlation between the electric field noise and the density. In the expanding solar wind where the density becomes low, the leaking of the spin period signal produces enhanced electric noise in low band channels. This effect is more significant for larger solar aspect angles (as in the second latitude scan, compared to the first scan), which produce stronger asymmetry of photoelectron cloud. This effect is also seen in the high band channels $(>9 \mathrm{~Hz})$ but is less significant. For example, if we express the relation between the electric wave power $\mathrm{P}_{\mathrm{E}}$ (in $\mathrm{V}^{2} / \mathrm{Hz}$ ) and the density $\mathrm{N}\left(\mathrm{cm}^{-3}\right)$ as $\log \mathrm{P}_{\mathrm{E}}=\mathrm{A}+\mathrm{B} \log \mathrm{N}$, for day 111-170 of year 2001 (the second latitude scan), for the low band channel at $5.33 \mathrm{~Hz}$, we have $\mathrm{B}=-0.33$, and the correlation coefficient, $\mathrm{C}$, between $\log \mathrm{P}_{\mathrm{E}}$ and $\log \mathrm{N}$ is as high as -0.74 ; while for the high band channel of 14 $\mathrm{Hz}, \mathrm{C}$ is only -0.35 . For the first scan with lower aspect angle, taking data from day 40 to 75 of 1995 , we have $\mathrm{C} \sim-0.35$ for $5.33 \mathrm{~Hz}$ channel, and $\mathrm{C} \sim 0.05$ for $14 \mathrm{~Hz}$ channel. Because of this photoelectron effect, the low band data is only useable for intense events. This problem was not addressed in paper (1). We will present a more detailed analysis in a future paper.

\section{ACKNOWLEDGMENTS}

The URAP experiment is a collaboration of NASA Goddard Space Flight Center, the Observatoire de Paris-Meudon, the University of Minnesota, and the Centre d'etude des Environnements Terrestres et Planetaires, Velizy, France. We thank the SWOOPS team and the Magnetometer team of Ulysses for the support of this work. N.L. thanks K. Goetz and S. J. Monson of the University of Minnesota for support in conducting this study.

\section{REFERENCES}

1. Lin, N., P. J. Kellogg, R. J. MacDowall, E. E. Scime, A. Balogh, R. J. Forsyth, D. J. McComas, and J. L. Philips, Very low frequency waves in the heliosphere: Ulysses observations, J. Geophys. Res., 103, 12,023 (1998).

2. MacDowall, R. J., and P. J. Kellogg, "Waves and instabilities in the 3-D heliosphere", in The Heliosphere Near Solar Minimum: The Ulysses Perspective, edited by A. Balogh, R. G. Marsden, E. J. Smith, London, Springer-Praxis, 2001, pp. 229-258.

3. Gary, S. P., E. E. Scime, J. L. Phillips, and W. C. Feldman, The whistler heat flux instability: Threshold conditions in the solar wind, J. Geophys. Res., 99, 23,391 (1994).

4. Scime, E. E., J. E. Littleton, S. P. Gary, and N. Lin, Solar cycle variations in the electron heat flux: Ulysses observations, Geophys. Res. Lett. 28, 2,169 (2001).

5. McComas, D. J., B. Goldstein, J. T. Gosling and R. M. Skoug, Ulysses' second orbit: Remarkably different solar wind, Space Sci. Rev. 97, 99 (2001). 